Check for updates

Cite this: Phys. Chem. Chem. Phys., 2022, 24, 786

Received 11th October 2021, Accepted 9th December 2021 DOI: $10.1039 / \mathrm{d} 1 \mathrm{cp04666g}$ rsc.li/pccp

\title{
Single photon double and triple ionization of allene $\dagger$
}

\author{
Veronica Ideböhn, ${ }^{a}$ Alistair J. Sterling, (D) ${ }^{\mathrm{b}}$ Måns Wallner, ${ }^{\mathrm{a}}$ Emelie Olsson, ${ }^{a}$ \\ Richard J. Squibb, ${ }^{a}$ Ugne Miniotaite, ${ }^{c}$ Emma Forsmalm, ${ }^{a}$ Malin Forsmalm, ${ }^{a}$ \\ Stefano Stranges, (D) de John M. Dyke, (D) Fernanda Duarte, (D) ${ }^{\mathrm{b}}$ \\ John H. D. Eland ${ }^{9}$ and Raimund Feifel (D) *a
}

\begin{abstract}
Double and triple ionization of allene are investigated using electron-electron, ion-ion, electronelectron-ion and electron-electron-ion-ion (ee, ii, eei, eeii) coincidence spectroscopies at selected photon energies. The results provide supporting evidence for a previously proposed roaming mechanism in $\mathrm{H}_{3}{ }^{+}$formation by double ionization. The lowest vertical double ionization energy is found to be $27.9 \mathrm{eV}$, while adiabatic double ionization is not accessed by vertical ionization at the neutral geometry. The triple ionization energy is found to be close to $50 \mathrm{eV}$ in agreement with theoretical predictions. The doubly charged parent ion is stable up to about $2 \mathrm{eV}$ above the threshold, after which dissociations by charge separation and by double charge retention occur with comparable intensities. Fragmentation to $\mathrm{H}^{+}+\mathrm{C}_{3} \mathrm{H}_{3}{ }^{+}$starts immediately above the threshold as a slow (metastable) decay with $130.5 \pm 9.9 \mathrm{~ns}$ mean lifetime.
\end{abstract}

\section{Introduction}

Allene (propadiene, $\mathrm{C}_{3} \mathrm{H}_{4}, \mathrm{CH}_{2}=\mathrm{C}=\mathrm{CH}_{2}$ ) is the prototypical and first member of the group of molecules called cumulenes. It is thought to be present in the interstellar medium ${ }^{1,2}$ at least as an intermediate, but as it lacks a dipole moment it has not yet been detected there directly. It is closely related to the important transient molecule ketene $\left(\mathrm{CH}_{2}=\mathrm{C}=\mathrm{CO}\right)$ which has been detected in the interstellar medium. ${ }^{3}$ This paper will report on single-photon double and triple ionization of allene, as well as on allene's dissociation pathways.

The single ionization of allene and its consequent dissociations have been studied using mass spectrometry, ${ }^{4}$ photoelectron

\footnotetext{
${ }^{a}$ Department of Physics, University of Gothenburg, Origovägen $6 B$,

41258 Gothenburg, Sweden. E-mail: raimund.feifel@physics.gu.se

${ }^{b}$ Department of Chemistry, Chemistry Research Laboratory, Mansfield Road, Oxford OX1 3TA, UK

${ }^{c}$ Department of Physics, Chalmers University of Technology, Kemigården 1, 41296 Gothenburg, Sweden

${ }^{d}$ IOM-CNR Tasc, SS-14, Km 163.5 Area Science Park, Basovizza 34149, Trieste, Italy

${ }^{e}$ Dipartimento di Chimica e Tecnologie del Farmaco, Universitá Sapienza, Rome I-00185, Italy

${ }^{f}$ School of Chemistry, University of Southampton, Highfield, Southampton SO17 1BJ, UK

${ }^{g}$ Department of Chemistry, Physical and Theoretical Chemistry Laboratory, Oxford University, South Parks Road, Oxford OX1 3QZ, UK

$\dagger$ Electronic supplementary information (ESI) available. See DOI: 10.1039/ d1cp04666g
}

spectroscopy ${ }^{5-7}$ and photoelectron-photoion coincidence spectroscopy. ${ }^{8}$ The electronic structure of the singly charged molecule has recently attracted theoretical interest, focusing on "Möbius" and "helical" frontier orbitals peculiar to cumulenes. ${ }^{9-11}$

Much less is known about multiple ionization of allene, but double and triple ionization of the molecule have both been examined in experiments using the electron capture charge exchange technique, ${ }^{12,13}$ where the lowest vertical double ionization energy to the expected ground-state triplet dication was determined as $28.2 \pm 0.3 \mathrm{eV}$. Allene double ionization and the rates of its dissociation reactions have also been examined using multi photon ionization with femtosecond infrared (IR) laser pulses. ${ }^{14,15}$ In these and other previous studies of allene double ionization, dissociation producing $\mathrm{H}_{3}{ }^{+}$ions has been found, surprisingly, to be more abundant than from molecules where three hydrogen atoms are initially contiguous in $-\mathrm{CH}_{3}$ groups. The mechanism was interpreted in the cases of $\mathrm{H}_{3}{ }^{+}$ formation from a series of hydrocarbon molecules with no methyl group, including allene, as involving a "roaming", mechanism where a neutral $\mathrm{H}_{2}$ is partially detached from the residual $\mathrm{C}_{3} \mathrm{H}_{2}{ }^{2+}$ fragment, after which a proton is transferred from the fragment dication to form the $\mathrm{H}_{3}{ }^{+}$ion, ${ }^{16,17}$ in line with the theoretical work of Mebel and Bandrauk. ${ }^{18}$ The creation of $\mathrm{H}_{3}{ }^{+}$is found to be a significant dissociation mechanisms for doubly charged allene, and must at minimum include the migration of one hydrogen atom ${ }^{14,18}$ and possibly more extensive rearrangement. A number of experiments probing this 
mechanism, mainly by IR multi-photon ionization in different molecules (mostly methanol) followed. ${ }^{19}$ Calculations on methanol indicate that the formation of $\mathrm{H}_{3}{ }^{+}$competes with that of $\mathrm{H}_{2}{ }^{+}$on a sub-100 fs time scale. ${ }^{19}$ In the present work we examine both $\mathrm{H}_{2}{ }^{+}$and $\mathrm{H}_{3}{ }^{+}$detected in correlation with the electrons involved in the creation of the nascent allene dications which dissociate to form them.

Our study was carried out using electron-electron (ee) coincidence measurements at photon energies above and below the C1s ionization thresholds (approximately $291 \mathrm{eV}$ ), and electron-electron-ion (eei) as well as electron-electron-ionion (eeii) coincidence measurements at $40.8 \mathrm{eV}$ photon energy. These measurements determine the spectra of states of nascent doubly ionised allene, and the spectra coincident with undissociated parent dications as well as each set of dissociation products. Photoionization mass spectra were also measured at all photon energies and ion-ion coincidence maps were taken to identify the decay pathways and clarify the mechanisms. The spectra and dissociation pathways are discussed and interpreted with the help of quantum chemical calculations carried out by ourselves. They are also compared with calculations available in the literature. ${ }^{18}$

\section{Experimental methods}

The experiments were carried out in our laboratory at the University of Gothenburg and at the synchrotron radiation facility BESSY-II of the Helmholtz Zentrum für Materialien in Berlin. In both cases, the target gas was let into the spectrometer using a hollow needle to create an effusive gas beam in the interaction region. The basic configuration of our system, which builds on a more compact version of the original magnetic bottle electronic spectrometet (MBES) instrument of this type,$^{20}$ comprises a strong conical magnet with a divergent field of approximately $1 \mathrm{~T}$ at the light-matter interaction point and a $2 \mathrm{~m}$ flight tube surrounded by a weak homogeneous solenoid field (few mT). At the end of the flight tube, electrons are registered by a multi-channel plate (MCP) detector, making the overall collection-detection efficiency of this MBES for low energy (<ca. $400 \mathrm{eV}$ ) electrons about 50-60\%. The electron energy resolution of the set-up is $E_{\text {kin }} / \Delta E_{\text {kin }} \sim 50$ when collecting only electrons. For collection of both electrons and ions the strong conical magnet is replaced by a hollow ring magnet with a weaker magnetic field in the interaction zone, which limits the electron energy resolution to $E_{\text {kin }} / \Delta E_{\text {kin }} \sim 20$, but with the benefit that ions can be extracted in the opposite direction. ${ }^{21,22}$ In this latter configuration, electron-ion coincidence data are obtained by first letting the electrons leave the interaction region, before accelerating the ions in the opposite direction, through the ring magnet and into a time of flight mass spectrometer with a $0.12 \mathrm{~m}$ long flight tube. The electric fields are optimized to achieve time focus conditions, giving a numerical resolving power for thermal ions of about 25. Under these conditions peaks in the parent ion group are only partially resolved. Complementary non-pulsed ion-only measurements were carried out with the magnetic bottle in its basic configuration by using the $2 \mathrm{~m}$ long flight tube for ions instead of electrons, and similar measurements were made using the shorter flight tube to capture processes on shorter time scales. With the longer flight path the numerical mass resolving power, $m / \Delta m$, was about 50, sufficient to resolve all the main ion peaks.

In the Gothenburg laboratory, a pulsed helium gas discharge lamp with a repetition rate of approximately $4 \mathrm{kHz}$ was used as ionization source where the atomic emission lines of HeI $\alpha$ and HeII $\alpha$ provided photon energies of 21.2 and $40.8 \mathrm{eV}$, respectively. The discrete energies were selected using a monochromator based on an ellipsoidal grating of 550 lines $\mathrm{mm}^{-1}$ groove density which also focuses the radiation of a selected wavelength into the interaction region.

The flight times of the electrons were converted to kinetic energies on the basis of a calibration derived from measurements of known photoelectron and Auger electron spectra. For calibration in the low electron kinetic energy region we used the photoelectron spectra of molecular oxygen at 21.2 and $40.8 \mathrm{eV}$ photon energy, and for higher energies the spectra of atomic argon and krypton at photon energies of $100 \mathrm{eV}$ and above.

At BESSY-II, the experimental set-up was mounted on undulator beamline UE52/SGM where the photon energy can be tuned in the extreme ultraviolet and soft X-ray energy region. In order to doubly ionise allene through valence-valence, coreAuger and core-valence electron removal photon energies of $100 \mathrm{eV}, 110 \mathrm{eV}, 300.5 \mathrm{eV}$ and $350.4 \mathrm{eV}$ were used. The set up was essentially the same as in Gothenburg without the He lamp but with the addition of a mechanical chopper synchronized to the radio frequency signal of the storage ring operating in single bunch mode. ${ }^{23}$ The chopper was set to increase the time interval between photon bunches passed to the experiment (otherwise $800.5 \mathrm{~ns}$ ) to between 10 and $100 \mu \mathrm{s}$, to allow detection of electrons and ions without interference from subsequent radiation pulses during their expected maximum flight times. The stated purity of the sample was $\geq 95 \%$. This was verified by on-line valence and core level photoelectron spectroscopy and mass spectroscopy which showed no impurity lines.

\section{Theoretical methods}

Allene in its neutral form, $\mathrm{C}_{3} \mathrm{H}_{4}$, has 22 electrons $\left(3 \times 1 \mathrm{~s}^{2} 2 \mathrm{~s}^{2} 2 \mathrm{p}^{2}\right.$ from the $\mathrm{C}$ atoms, $4 \times 1 \mathrm{~s}^{1}$ from the $\mathrm{H}$ atoms) and its ground state has a $D_{2 \mathrm{~d}}$ geometry. The outermost electronic configuration is $-3 \mathrm{~b}_{2}{ }^{2} 1 \mathrm{e}^{4} 2 \mathrm{e}^{4}$, which, in a simplified picture, can be viewed as derived from the $2 \mathrm{p}^{2}$ electrons of each carbon and the $1 \mathrm{~s}^{1}$ electrons of each $\mathrm{H}$ atom. ${ }^{24}$ The $2 \mathrm{e}$ and $1 \mathrm{e}$ orbitals are $\pi$ orbitals and the $3 b_{2}$ orbital is a $\sigma$ orbital. Removal of two electrons from the outermost $2 \mathrm{e}$ orbital gives rise to four states: ${ }^{3} \mathrm{~A}_{2},{ }^{1} \mathrm{~A}_{1},{ }^{1} \mathrm{~B}_{1}$ and ${ }^{1} \mathrm{~B}_{2}$. The lowest energy singlet state $\left({ }^{1} \mathrm{~A}_{1}\right)$ of allene $^{2+}$ undergoes a geometric distortion $\left(D_{2 \mathrm{~d}} \rightarrow D_{2 \mathrm{~h}}\right)$ to remove orbital degeneracy (see Fig. 1 and ESI $\dagger$ ). Vertical ionization energies to these $(2 \mathrm{e})^{-2}$ states were calculated as the difference in total energies of the neutral and dicationic states 


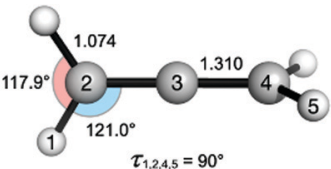

$\mathrm{D}_{2 \mathrm{~d}} \mathrm{C}_{3} \mathrm{H}_{4}\left({ }^{1} \mathrm{~A}_{1}\right)$

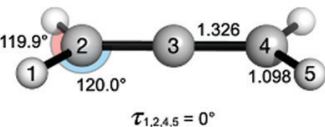

$\mathrm{D}_{2 \mathrm{~h}} \mathrm{C}_{3} \mathrm{H}_{4}{ }^{2+}\left({ }^{1} \mathrm{Ag}_{\mathrm{g}}\right)$
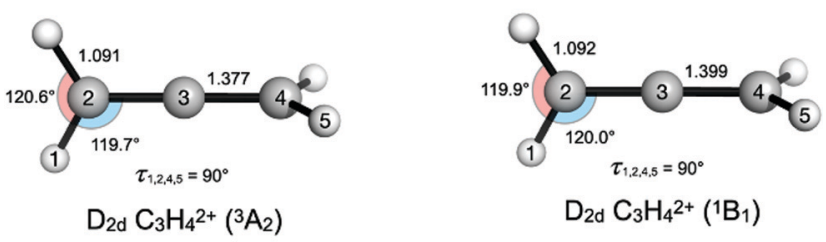

$\mathrm{D}_{2 \mathrm{~d}} \mathrm{C}_{3} \mathrm{H}_{4}{ }^{2+}\left({ }^{1} \mathrm{~B}_{1}\right)$

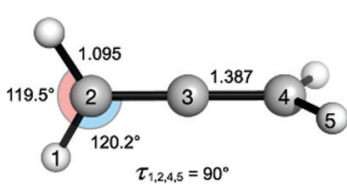

$\mathrm{D}_{2 \mathrm{~d}} \mathrm{C}_{3} \mathrm{H}_{4}{ }^{2+}\left({ }^{1} \mathrm{~B}_{2}\right)$

Fig. 1 Changes in electronic structure of neutral $D_{2 d}$ allene upon vertical double ionization (in eV) to the four lowest energy states to give: ${ }^{3} A_{2},{ }^{1} A_{1}$, ${ }^{1} B_{1}$ and ${ }^{1} B_{2}$ that make up the first peak in the double ionization spectrum in Fig. 2. The $D_{2 d}{ }^{1} A_{1}$ state undergoes a geometric distortion from $D_{2 d} \rightarrow D_{2 h}$ to remove orbital degeneracy. Optimised geometries at the CASSCF/ def2-TZVP level are shown, with distances in $\AA$ and angles in degrees.

at the optimised neutral ground state geometry of allene. Adiabatic ionization energies were calculated as the difference in the electronic energies of the neutral and dicationic states, each at their optimised geometry (see ESI $\dagger$ ).

All calculations were carried out using the ORCA program (v 4.1.2). ${ }^{25}$ Optimisations were carried out at the $\operatorname{CASSCF}(4,4)$ and CASSCF $(2,4)$ levels with the def2-TZVP basis $\operatorname{set}^{26}$ for neutral and doubly-ionized states (see ESI $\dagger$ ), respectively, reflecting ionization from the $2 \mathrm{e}(\pi)$ orbitals of allene. Optimisation used "tight" thresholds defined in ORCA, corresponding to an SCF energy convergence of $10^{-8} \mathrm{Ha}$. Ionization energies were obtained at the NEVPT2 level, ${ }^{27}$ based on $\operatorname{CASSCF}(4,4)$ and CASSCF $(2,4)$ wavefunctions for neutral and doubly-ionized states, respectively, using the def2-QZVPP basis set (see ESI $\dagger$ ). Static correlation arising from (near)-degeneracy, that is often a challenge for single-reference methods, ${ }^{12}$ is explicitly accounted for using the current procedure, and dynamic correlation is included by the NEVPT2 calculations.

\section{Results and discussion}

\subsection{Double ionization of Allene}

4.1.1 Valence double ionization. Fig. 2 shows double ionization spectra from electron-electron coincidence measurements at photon energies of 40.8 and $100 \mathrm{eV}$. Accidental coincidences have been subtracted. The two spectra agree well with each other, reflecting the onset of double ionization at about $27 \mathrm{eV}$ as part of the first band with a vertical double ionization energy of about $27.9 \mathrm{eV}$, the latter in very good

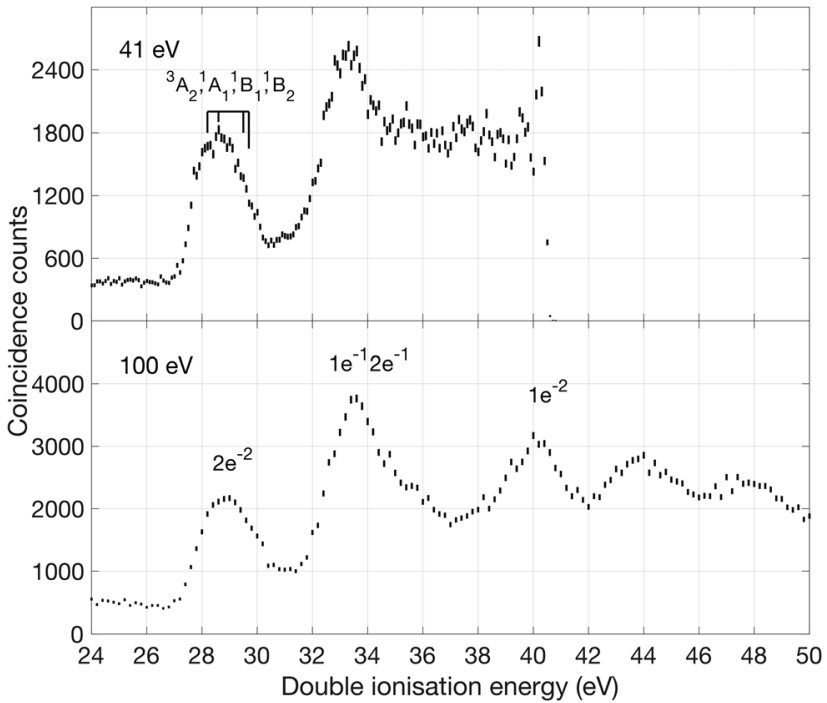

Fig. 2 Double ionization electron spectra of allene measured at the photon energies of $40.8 \mathrm{eV}$ (upper panel) and $100 \mathrm{eV}$ (lower panel). The $40.8 \mathrm{eV}$ data were taken in Gothenburg with a helium gas discharge lamp and the $100 \mathrm{eV}$ data were taken at BESSY-II in Berlin. Both spectra are based on electron pair measurements. Assignments in terms of leading configuration (lower panel), and for the lowest structure in the $40.8 \mathrm{eV}$ spectrum in terms of different electronic states (upper panel) are included.

agreement with the theoretical value of $28.2 \mathrm{eV}$. Additional structures appear above $30 \mathrm{eV}$ double ionization energy, which are centred near 33.5, 36, 40, 44 and $48 \mathrm{eV}$ and which involve removing more tightly bound electrons in allene. The bands associated with electron removal from the degenerate orbitals are expected to be the most intense because of the larger number of electrons in them, but they probably conceal underlying bands from combinations with the other ( $\sigma$-type) orbitals.

The first band is especially interesting, with substructures at $27.9,28.7,29.3$ and $30.0 \mathrm{eV}$ that are found by fitting the data to four separate Gaussian peaks, as described in the ESI. $\dagger$ The peaks are interpreted with the aid of our calculations of vertical ionization energies to the lowest triplet $\mathrm{T}_{0}\left({ }^{3} \mathrm{~A}_{2}\right.$ in $\left.D_{2 \mathrm{~d}}\right)$ state, the lowest singlet $S_{0}$ state, which undergoes reorganization, possibly to ${ }^{1} \mathrm{~A}_{\mathrm{g}}$ in $D_{2 \mathrm{~h}}$ or to a lower symmetry structure, and the second and third singlet states $\left({ }^{1} \mathrm{~B}_{1}\right.$ and $\left.{ }^{1} \mathrm{~B}_{2}\right)$ which retain $D_{2 \mathrm{~d}}$ geometry, as illustrated in Fig. 1. Because of the resolution of approximately $0.3 \mathrm{eV}$ in the energy area of the first peak, we are not able to fully resolve the individual peaks, but by fitting the data to four Gaussian peaks the individual peak values were obtained. The peak position from the fit seem to agree with theory and previous measurements of double ionization of allene. $^{12,13}$

Our theoretical values for the double ionization energy of the different states as well as the experimental values taken from Fig. 2, are summarized in Table 1. The theoretical and experimental values are very close to each other, but adiabatic ionization to the rearranged ground state is not observed. Our calculations are in excellent agreement with the double ionization calculations by Andrews et al. at the MP2 and MP4 levels of theory. ${ }^{12,13}$ In the double ionization of allene, the molecule 
Table 1 Theoretical values for the vertical and adiabatic double ionization of allene, in eV, from calculations carried out here using NEVPT2/CASSCF(4,4) and NEVPT2/CASSCF $(2,4)$ for neutral and doubly-ionized states (def2-QZVPP basis set), respectively, compared with previous values from Andrews et al. ${ }^{12,13}$ (in parentheses, MSX- $\alpha$ level), Wong and Radom ${ }^{28}$ [in square brackets, MP4/6-311G*//MP2/6-31G* level], and Mebel and Bandrauk ${ }^{18}$ \{curly brackets, CCSD(T)/CBS//B3LYP/6-31G* level\}. For comparison, experimental values extracted from Fig. 1 are also included. The experimental values have been found through fit of Gaussian functions to the entire peak

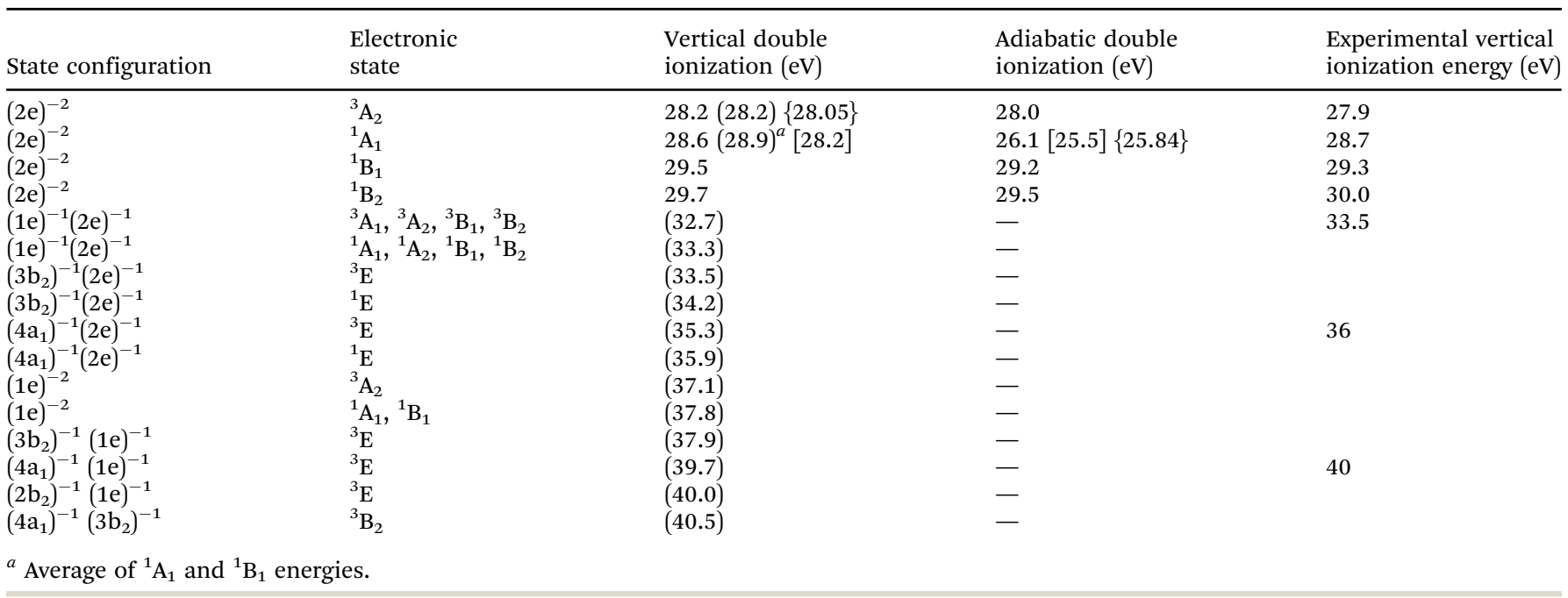

goes from being described by the point group $D_{2 \mathrm{~d}}$ to $D_{2 \mathrm{~h}}$ through a reorganisation of the orbitals and a reduction of degenerate states. This process, where the 3D structure of neutral allene changes to a planar form upon double ionization is schematically illustrated in Fig. 1. Although their calculations are otherwise very extensive, Mebel and Bandrauk did not report calculated energies or structures for $\mathrm{S}_{1}$ or any higher states of doubly ionised allene. ${ }^{18}$

The lowest (adiabatic) double ionization energy is calculated in the present work as $26.1 \mathrm{eV}$, which is somewhat higher than the previously reported value of $25.8 \mathrm{eV}$ (see Table 1 of ref. 18), but still well below the range seen to be accessed by singlephoton ionization in our spectra. The triplet state also relaxes, but much less, ending up at an adiabatic double ionization energy of $28.0 \mathrm{eV}$, which is in the range seen in our spectrum.

4.1.2 Double ionization by Auger decay. Electronic states with two vacancies in valence orbitals can also be formed by initial inner shell (C1s) ionization followed by emission of a secondary Auger electron on filling of the short-lived core hole. In the case of allene, single ionization from the C1s orbitals was reported by Travnikova et al. to occur at 290.6 and $290.8 \mathrm{eV}$, giving rise to photoelectron lines that were only partially resolved in our electron pair measurements carried out at $300.5 \mathrm{eV}$ photon energy ${ }^{29}$ In analyzing the coincidence data, partial separate selection is possible by choosing the extreme sides of the composite photoelectron line feature. Double ionization electron spectra (incorporating the energy of the selected photoelectrons) obtained in this way are shown in Fig. 3.

As can be seen, there is a clear difference between the two selectively extracted spectra. Also, even though the experimental resolution is limited ( $c a .5 \mathrm{eV}$ ) because of the high electron kinetic energies, substructures that correspond well with the distinct bands seen in the $100 \mathrm{eV}$ spectra ( $c f$. Fig. 2)

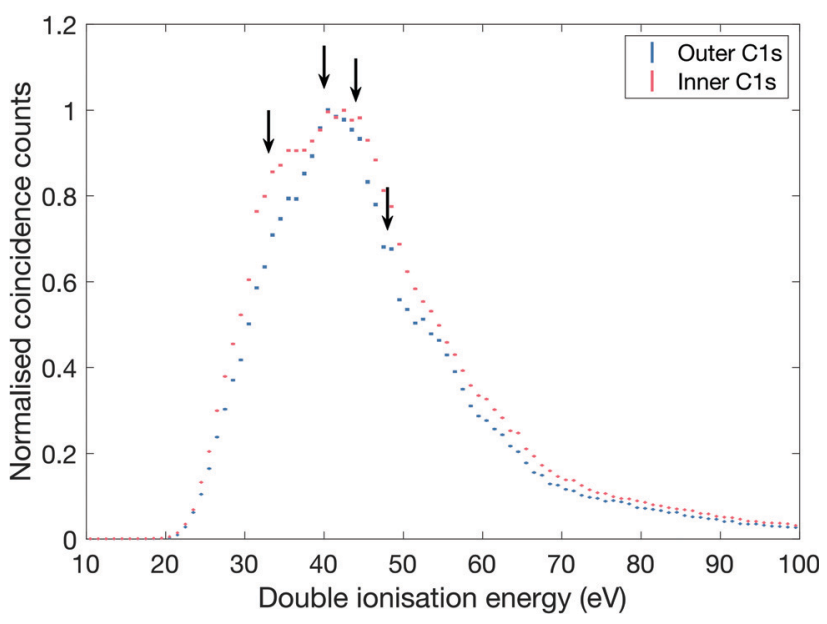

Fig. 3 Double ionization spectra of allene measured with $300.5 \mathrm{eV}$ photon energy. The spectra have been selected on outer and inner $\mathrm{C} 1 \mathrm{~s}$ core electron, respectively. The inner C1s electron has a binding energy of $290.8 \mathrm{eV}$ and the outer C1s electron has $290.6 \mathrm{eV}^{29}$ and the selections comprise binding energy ranges of $\pm 0.1 \mathrm{eV}$. The arrows indicate structures in the ionization spectra at energies of about $33,40,44$ and $48 \mathrm{eV}$.

are discernible as indicated by arrows, at about $33,40,44$ and $48 \mathrm{eV}$.

\subsection{Triple ionization of allene}

Triple ionization of allene can occur by different main pathways, according to the photon energy. At $100 \mathrm{eV}$ there is only triple valence electron removal. At all energies above about $300 \mathrm{eV}$ core ionization and subsequent double Auger decay is dominant, while at and above $330 \mathrm{eV}$ there is also corevalence direct double ionization followed by Auger decay. Spectra corresponding to the three different pathways are shown in Fig. 4. 


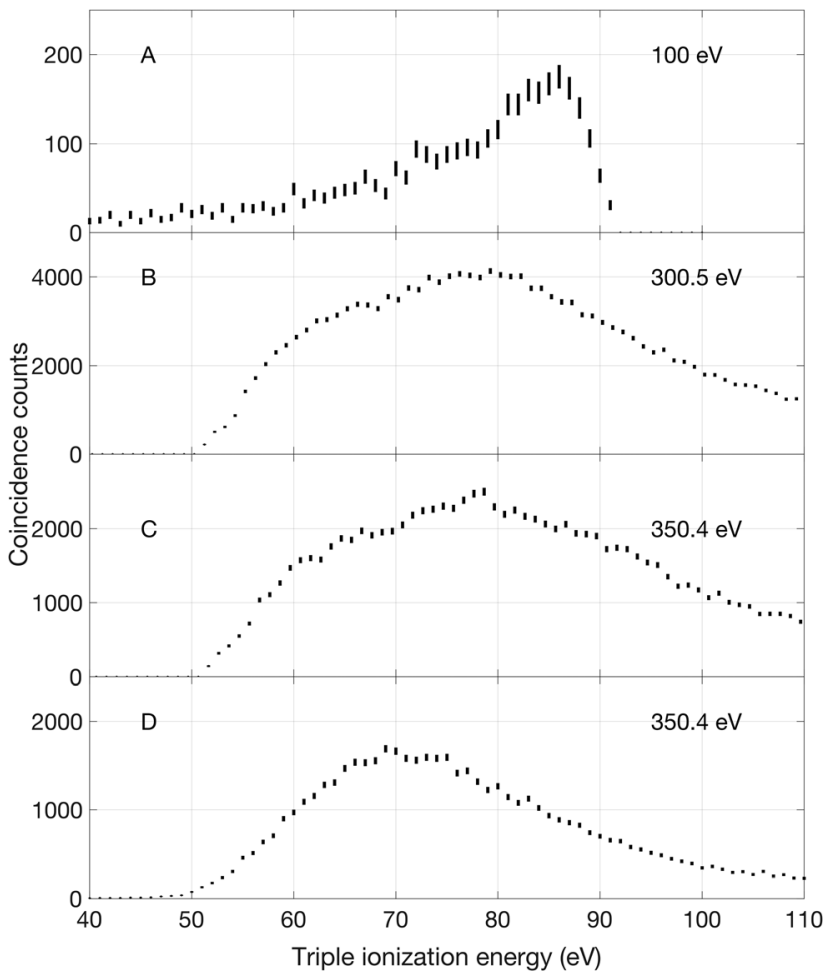

Fig. 4 Triple ionization at 100, 300.5 and $350.4 \mathrm{eV}$ photon energies. In panel $A$, the triple ionization spectrum has been extracted by limiting the data to events where the slowest electron is within the energy range of $3 \pm 0.5 \mathrm{eV}$. In panels B and C, events with one core electron emission and two other electron emissions have been selected. The triple ionization spectrum in panel $D$ is derived by identifying events where a core-valence doubly ionised state is formed before an Auger electron is emitted.

The $100 \mathrm{eV}$ spectrum (panel A in Fig. 4) is essentially structureless, showing only a smoothly rising signal with a possible start at about $50 \mathrm{eV}$ (and an uncertainty of nearly $2 \mathrm{eV}$ ). To remove spurious low energy electrons, the lowest energy included in the events was set to $3 \pm 0.5 \mathrm{eV}$.

The triple ionization spectra displayed in panels $\mathrm{B}$ and $\mathrm{C}$ were extracted from measurements at 300.5 and $350.4 \mathrm{eV}$ photon energy by selecting events with one core electron and two other electrons. These selections excluded electrons that were not part of the desired energy sharing. As can be seen, the spectra reflect a broad, featureless bump with onset at about $51 \pm 2.3 \mathrm{eV}$ and maximum intensity in the 79-80 eV energy range. We note that the ratio of double to single Auger decay is experimentally found to be $13 \%$ at both $300.5 \mathrm{eV}$ and $350.4 \mathrm{eV}$ photon energy which is slightly higher than reported ratios in other $\mathrm{C}_{3}$ carbon compounds from the work of Hult Roos et $a .^{30}$

At $350.4 \mathrm{eV}$ we could also extract the triple ionization spectrum shown in panel $\mathrm{D}$, based on selection of initial core-valence double ionization, for which full spectra will be presented in a forthcoming publication, followed by an additional Auger electron emission. The spectrum locates the lowest triple ionization energy at about $50 \pm 5.4 \mathrm{eV}$. All these onset values are in reasonable agreement with the calculated adiabatic triple ionization values given by Mebel and Bandrauk as $52.29 \mathrm{eV}^{18}$

\subsection{Fate of Allene above lowest double ionization threshold}

To investigate the fate of allene exposed to photon energies above the lowest double ionization threshold ion detection in multiplex was employed.

4.3.1 Ion time-of-flight mass spectra. Fig. 5 shows mass spectra of allene obtained at the photon energies of $100 \mathrm{eV}$ (upper panel) and $300.5 \mathrm{eV}$ (lower panel). Both spectra were obtained using the $2 \mathrm{~m}$ flight tube for ion detection. The $100 \mathrm{eV}$ spectrum is very similar to the mass spectrum at $40.8 \mathrm{eV}$ which is not shown separately.

As can be seen, the $100 \mathrm{eV}$ spectrum is dominated by the parent ion group $(m / q=40,39,38,37,36)$. The weak and otherwise unexpected peak(s) at 32 (and 28) suggest(s) that a small amount of air was present during this recording. More interestingly, there are relatively strong peaks for doubly charged ions containing the $\mathrm{C}_{3}$ species at $m / q=20,19.5$ and 19 , all three of roughly equal intensity. The very weak peak at $m / q=16$ is probably due to $\mathrm{O}^{+}$, while the peak at $m / q=15$ of similar intensity is attributed to the $\mathrm{CH}_{3}{ }^{+}$species. Its intensity is about $1 / 4$ that of the $m / q=14$ peak associated with $\mathrm{CH}_{2}{ }^{+}$. Very interestingly, there are distinct peaks for $m / q=2$ and 3, i.e. for $\mathrm{H}_{2}{ }^{+}$and $\mathrm{H}_{3}{ }^{+}$. The remainder of the peaks will be discussed later.

The most striking points in comparing the mass spectrum at $300.5 \mathrm{eV}$ with the $100 \mathrm{eV}$ data are the greatly increased dissociation at $300.5 \mathrm{eV}$ where double ionization by the Auger effect dominates, and the observed stability of the doublycharged ions $\mathrm{C}_{3} \mathrm{H}_{4}{ }^{2+}, \mathrm{C}_{3} \mathrm{H}_{3}{ }^{2+}$ and $\mathrm{C}_{3} \mathrm{H}_{2}{ }^{2+}$ (but not $\mathrm{C}_{3} \mathrm{H}^{2+}$ or

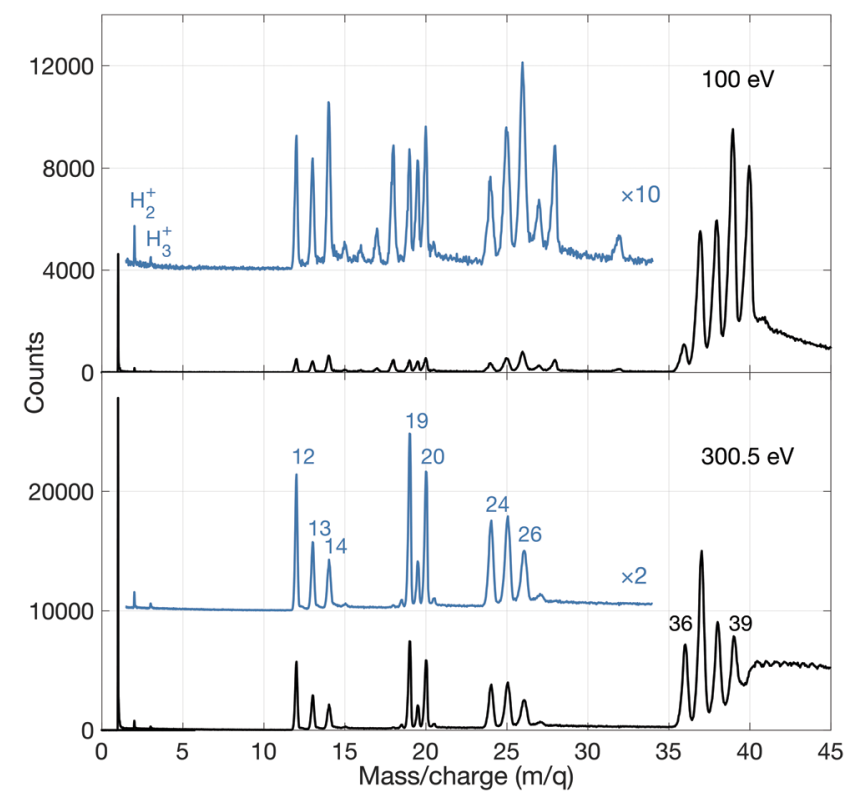

Fig. 5 Mass spectra of allene at photon energies $100 \mathrm{eV}$ (upper panel) and $300.5 \mathrm{eV}$ (lower panel). The mass spectrum at $40.8 \mathrm{eV}$ (not shown) is very similar to the $100 \mathrm{eV}$ spectrum. The blue spectra are multiplied by a factor of 10 and 2 in the upper and lower panel, respectively. The numbers refer to the mass over charge assigned to the specific peaks. 
$\mathrm{C}_{3}{ }^{2+}$ ). We note that the structure and energetics of the parent doubly charged ion were calculated by Mebel and Bandrauk, but those of $\mathrm{C}_{3} \mathrm{H}_{2}{ }^{2+}$ and $\mathrm{C}_{3} \mathrm{H}^{2+}$ were not. ${ }^{18}$ They, and the apparently less stable or unstable $\mathrm{C}_{3} \mathrm{H}^{2+}$ and $\mathrm{C}_{3}{ }^{2+}$ species are fundamental entities, likely to be of relevance in astrophysical contexts. From a theoretical point of view, not much is known about their structure, except for the $\mathrm{C}_{3}{ }^{2+}$ species for which a linear configuration has been suggested. ${ }^{31}$ However, for $\mathrm{C}_{3}{ }^{+}$ there is experimental evidence from Coulomb explosion imaging $^{32}$ and theory ${ }^{33,34}$ that it is non-linear.

4.3.2 Ion-ion coincidences. An ion-ion coincidence map associated with the $100 \mathrm{eV}$ ion time-of-flight spectrum from Fig. 5 and after subtraction of accidental coincidences is shown in Fig. 6. The detectable ion pairs seen in this map are summarized in Table 2.

Apart from the numerous ion pairs observed at $100 \mathrm{eV}$, some of which may come from triple ionization and involve an unobserved third ion, four double-charge-retaining dissociation channels producing $\mathrm{C}_{3} \mathrm{H}_{3}{ }^{2+}, \mathrm{C}_{3} \mathrm{H}_{2}{ }^{2+}, \mathrm{C}_{3} \mathrm{H}_{2}{ }^{2+}$ and $\mathrm{C}_{3}{ }^{2+}$, as seen in Fig. 5, arise entirely from double ionization. From these and other data we identify the $\mathrm{H}^{+}+\mathrm{C}_{3} \mathrm{H}^{+}+\mathrm{H}_{2}$ double ionization channel as the most probable charge separation channel. As can be seen in Fig. 5, the number of charge retaining products increase with increased photon energy. For thermal ions the efficiency measured in separate experiments was about $7 \%$, but the actual efficiency for charge-separating ion pairs will be lower because of losses caused by their initial kinetic energies. This means that the charge retaining products are overestimated if we only consider counts in Fig. 5 and are not one of the more abundant ionization channels.

Fig. 7 displays an ion-ion coincidence map of allene obtained at the photon energy of $40.8 \mathrm{eV}$ in DC mode using a much shorter (about $0.12 \mathrm{~m} \mathrm{long}$ ) ion time-of-flight spectrometer

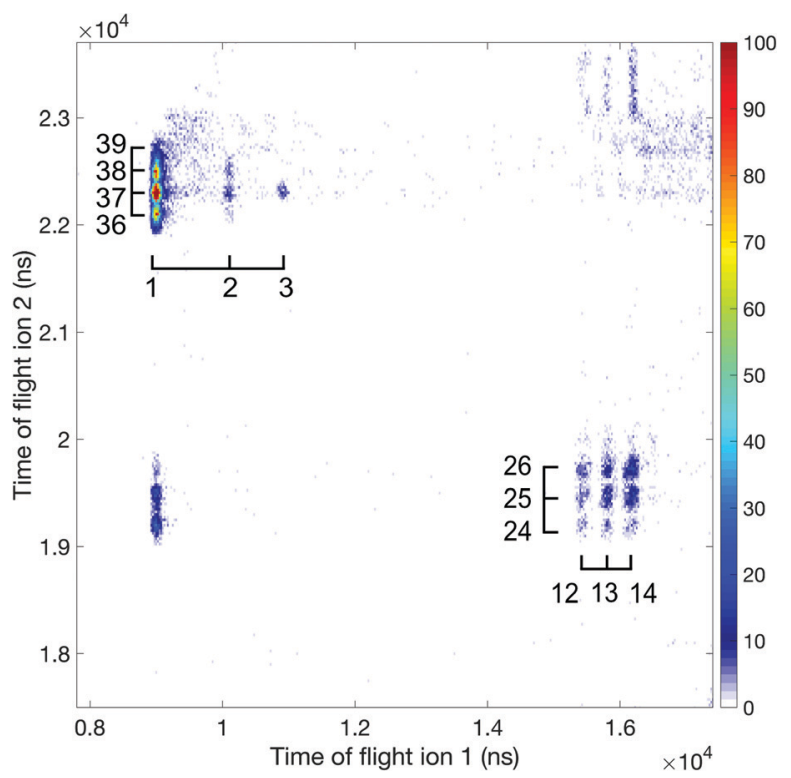

Fig. 6 Ion-ion coincidence map at $100 \mathrm{eV}$ photon energy using the $2 \mathrm{~m}$ flight tube. Accidental coincidences have been removed and the mass over charge information is included in the plot.
Table 2 lon pairs detected in the $100 \mathrm{eV}$ ion-ion coincidence map shown in Fig. 6 . Note: the $\mathrm{H}^{+}+\mathrm{CH}^{+}$or $\mathrm{C}^{+}$channels are not included in the table

\begin{tabular}{ll}
\hline Ion pair & Rel. abundance $(\Sigma=1000)$ \\
\hline $\mathrm{H}^{+}+\mathrm{C}_{3} \mathrm{H}_{3}^{+}$or $\mathrm{C}_{3} \mathrm{H}_{2}{ }^{+}$or $\mathrm{C}_{3} \mathrm{H}^{+}$or $\mathrm{C}_{3}^{+}$ & 562 \\
$\mathrm{H}^{+}+\mathrm{C}_{2} \mathrm{H}^{+}$or $\mathrm{C}_{2}^{+}$ & 92 \\
$\mathrm{H}^{+}+\mathrm{CH}^{+}$or $\mathrm{C}^{+}$ & 72 \\
$\mathrm{H}_{2}^{+}+\mathrm{C}_{3} \mathrm{H}_{2}^{+}$or $\mathrm{C}_{3} \mathrm{H}^{+}$or $\mathrm{C}_{3}{ }^{+}$ & 33 \\
$\mathrm{H}_{3}^{+}+\mathrm{C}_{3} \mathrm{H}^{+}$or $\mathrm{C}_{3}^{+}$ & 12 \\
$\mathrm{C}^{+}+\mathrm{C}_{2} \mathrm{H}_{3}^{+}$or $\mathrm{C}_{2} \mathrm{H}_{2}^{+}$or $\mathrm{C}_{2} \mathrm{H}^{+}$or $\mathrm{C}_{2}^{+}$ & 49 \\
$\mathrm{CH}^{+}+\mathrm{C}_{2} \mathrm{H}_{3}^{+}$or $\mathrm{C}_{2} \mathrm{H}_{2}{ }^{+}$or $\mathrm{C}_{2} \mathrm{H}^{+}$or $\mathrm{C}_{2}^{+}$ & 76 \\
$\mathrm{CH}_{2}^{+}+\mathrm{C}_{2} \mathrm{H}_{3}^{+}$or $\mathrm{C}_{2} \mathrm{H}_{2}^{+}$or $\mathrm{C}_{2} \mathrm{H}^{+}$or $\mathrm{C}_{2}^{+}$ & 104
\end{tabular}

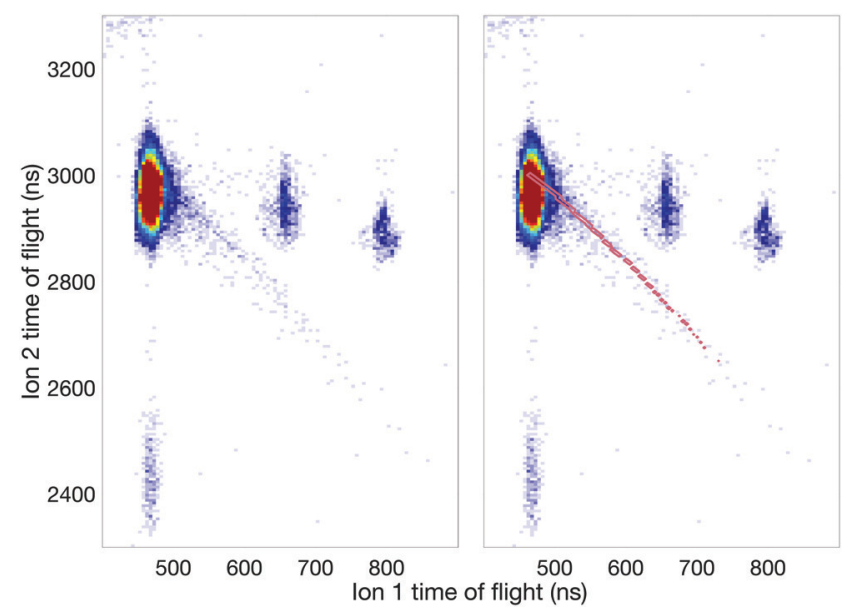

Fig. 7 Panel A displays an ion-ion coincidence map at $40.8 \mathrm{eV}$ photon energy using the shorter $(0.12 \mathrm{~m})$ flight tube. Mass/charge is denoted by the numbers in the figure. The sloping feature which starts at the correlation island of mass 1 and 39 and extends towards the lower right corner, indicates the presence of a metastable doubly charged state with a lifetime on the order of the flight times of the ions in this shorter tube. This metastable state dissociates into the two singly charged ions seen in the map, $\mathrm{H}^{+}$and $\mathrm{C}_{3} \mathrm{H}_{3}{ }^{+}$, as illustrated in panel $\mathrm{B}$ by the pink markings. The markings have been obtained by simulating the decay in SIMION with a lifetime of the metastable state of $130.5 \pm 9.9$ ns.

mounted in tandem configuration to the electron flight tube. This map confirms, though at lower resolution, several of the ion pair channels involving the $\mathrm{H}^{+}, \mathrm{H}_{2}{ }^{+}$, and $\mathrm{H}_{3}{ }^{+}$species. The significant appearance of $\mathrm{H}_{3}{ }^{+}$ions in coincidence is in agreement with the findings of Hoshina et al. ${ }^{14}$ and related theory. ${ }^{18}$ While the mechanism of formation of the $\mathrm{H}_{3}{ }^{+}$ions is discussed in the literature in terms of a "roaming" mechanism, which involves an initial $\mathrm{H}_{2}$ species that "orbits" within a doublycharged precursor until it succeeds in extracting the third proton, the identity of the electronic state from which it happens in allene dication has not been determined experimentally. According to the energy range it could be any of the four states in the first double ionization band ( $c f$. Fig. 2). If Mebel and Bandrauk's RRKM rate calculation is taken at face value, it means that $\mathrm{H}^{+}+\mathrm{C}_{3} \mathrm{H}_{3}{ }^{+}$come from the relaxed ground state $\mathrm{S}_{0} \cdot{ }^{18}$ Further support for that can be obtained from Fig. 3 of Mebel and Bandrauk ${ }^{18}$ according to which the ground state can make hiving off "roaming $\mathrm{H}_{2}$ " more likely by the involvement of 
a methyl acetylene transition state which posses favourable structural characteristics.

The most interesting aspect in the $40.8 \mathrm{eV}$ map is the presence of an unambiguous, though weak metastable tail, which is essentially invisible at $100 \mathrm{eV}$ and higher photon energies. The map identifies the most intense part of the tail as belonging to the $\mathrm{H}^{+}+\mathrm{C}_{3} \mathrm{H}_{3}{ }^{+}$reaction channel but its comparatively broad spread may also include a contribution from the $\mathrm{H}^{+}+\mathrm{C}_{3} \mathrm{H}_{2}{ }^{+}$ion pairs. The $\mathrm{H}^{+}+\mathrm{c}_{-} \mathrm{C}_{3} \mathrm{H}_{3}{ }^{+}$(cyclic cyclopropenyl) ion pair has the lowest thermodynamic formation limit at $25 \mathrm{eV}$, while almost all the other observed pairs have limits near $26 \mathrm{eV}$ for formation with no kinetic or internal energy release. Since there is no detectable "V" shape from the metastable decay centred on the doubly charged parent ion's position as apex on the diagonal $\left(t_{1}=t_{2}\right),{ }^{35}$ no longer-lived group of ions dissociates in the flight tube. This implies that essentially all the metastable decay takes place in the source field of our spectrometer.

The existence of this metastable decay was already noted by Barber and Jennings in 1969 in electron impact mass spectrometry. ${ }^{36}$ To investigate the metastable lifetime, we plotted the coincidence data as a map of $t_{1}+t_{2} v s$. $t_{2}-t_{1}$, where the metastable tail gets concentrated as a strip almost parallel to the $\left(t_{2}-t_{1}\right)$-axis. The intensity as a function of the time difference was extracted and plotted, as is done in Fig. 8, for comparison with the theory given by Field and Eland. ${ }^{35}$ The fit to the metastable state in the figure has a 95\% confidence interval of 229.2-266.8 ns which translates to a lifetime of $130.5 \pm 9.9 \mathrm{~ns}$. The observation that the experimental points fit well to a single exponential decay with a mean lifetime of $130.5 \mathrm{~ns}$ is in contrast to most other cases, where metastable lifetime decay curves investigated in this way represent a mixture or wide distribution of lifetimes. ${ }^{35}$ This may imply that

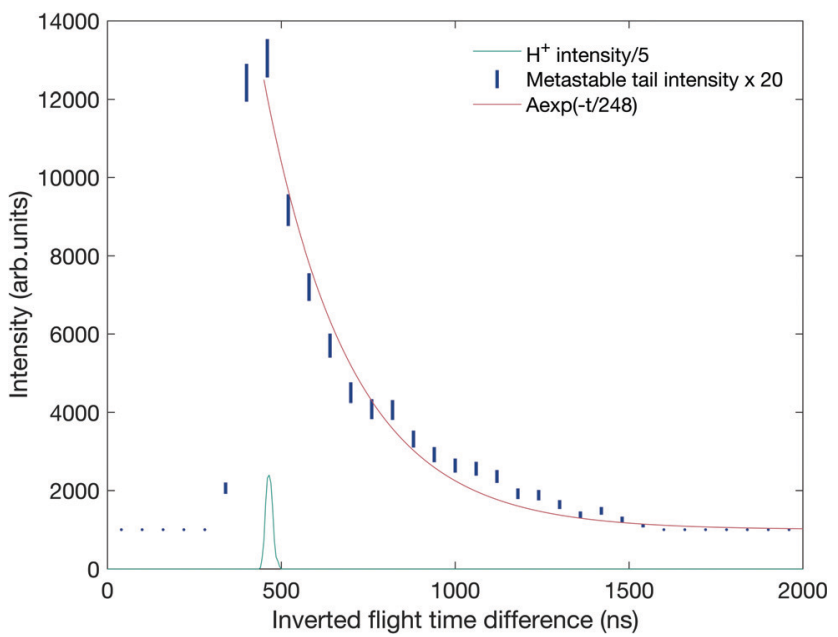

Fig. 8 Metastable tail intensity as a function of the inverted flight time difference with a fit to the exponential decay in red. The coincidences are the same as in the tail in Fig. 7 plotted against $t_{2}-t_{1}$ where $t_{1}$ and $t_{2}$ are the flight times of the first and second ion to be detected, respectively. The location of the mass peak for $\mathrm{H}^{+}$ions on the same scale is included below the decay curve for comparison. only one single vibration level or a very close group lies just above the barrier to this reaction channel.

Because significant approximations are involved in the theory behind the analysis of Fig. 8, the metastable decay was also investigated by a Monte-Carlo type simulation using a realistic model of the apparatus implemented in the software package SIMION, ${ }^{37}$ the results of which are presented in panel B of Fig. 7. The decay was modelled to have a $3 \mathrm{eV}$ kinetic energy release (KER) in line with the observed difference in energy between the thermochemical threshold and the observed appearance energy, reported below. The pink contour shows the simulation results on top of the experimental coincidence map. This simulation fits the experimental observations well with a mean lifetime of $130 \pm 10 \mathrm{~ns}$, in good agreement with the simple exponential fit. We note that this lifetime is within the range predicted by Mebel and Bandrauk on the basis of RRKM theory, that is, assuming free flow of internal energy within the molecular ion allowing statistical energy redistribution.

4.3.3 Multiple-electron-ion coincidences and action spectra. To determine the appearance energies of the different sets of products and to obtain "action spectra" for the different channels as a function of double ionization energy, threefold (eei) and fourfold (eeii) coincidences are indispensable. In view of the achievable electron energy resolution, this was primarily done at the photon energy of $40.8 \mathrm{eV}$. If a particular ion is formed by only one decay channel, an eei spectrum is sufficient to define the action spectrum. This is true for all three doubly charged ions $\mathrm{C}_{3} \mathrm{H}_{4}{ }^{2+}, \mathrm{C}_{3} \mathrm{H}_{3}{ }^{2+}$ and $\mathrm{C}_{3} \mathrm{H}_{2}{ }^{2+}$ for which the spectra are shown in Fig. 9. It is also true for ions formed only in two-body decays of the parent including the metastable decay channel shown in Fig. 7. Analysis of the ion-ion coincidence maps suggests that it applies as a good approximation to $\mathrm{H}_{3}{ }^{+}$formation (action spectrum in the upper panel of Fig. 10) and to the ionization pathways leading to $\mathrm{C}_{2} \mathrm{H}_{2}^{+}$or $\mathrm{CH}_{2}^{+}$and to $\mathrm{C}_{2} \mathrm{H}_{3}^{+}$or $\mathrm{CH}^{+}$whose spectra are shown in Fig. 11. The other ions, $\mathrm{H}^{+}, \mathrm{H}_{2}{ }^{+}$and $\mathrm{C}_{3} \mathrm{H}_{n}{ }^{+}$, are all products of more than one decay channel, so eeii coincidences are formally required to get unambiguous action spectra. Unfortunately, the ion collection efficiency of the apparatus is so low that spectra with useful numbers of counts cannot be obtained for most such fourfold coincidences so eei events have been used to generate the spectra shown in Fig. 10.

From these spectra, we can get an overview of the fates of allene dications in the range of the excitation energies covered by the double photoionization spectrum at $40.8 \mathrm{eV}$. As can be seen in Fig. 9, the first peak of the total double ionization spectrum is primarily associated with undissociated electronic states of the doubly ionised parent molecule at up to $3 \mathrm{eV}$ above the calculated adiabatic double ionization threshold of $26 \mathrm{eV}$. Because of an overlap in the mass spectrum of the peaks reflecting the parent ion and the parent ion minus one hydrogen atom as well as minus a hydrogen molecule, it was impossible to separate the signals for these species completely. We believe that the peak in the double ionization spectrum of $\mathrm{C}_{3} \mathrm{H}_{3}{ }^{2+}$ between 26 and $30 \mathrm{eV}$ actually originates from $\mathrm{C}_{3} \mathrm{H}_{4}{ }^{2+}$ instead, because otherwise its appearance energy would be impossibly low. Also, the high energy part of the $\mathrm{C}_{3} \mathrm{H}_{4}{ }^{2+}$ 


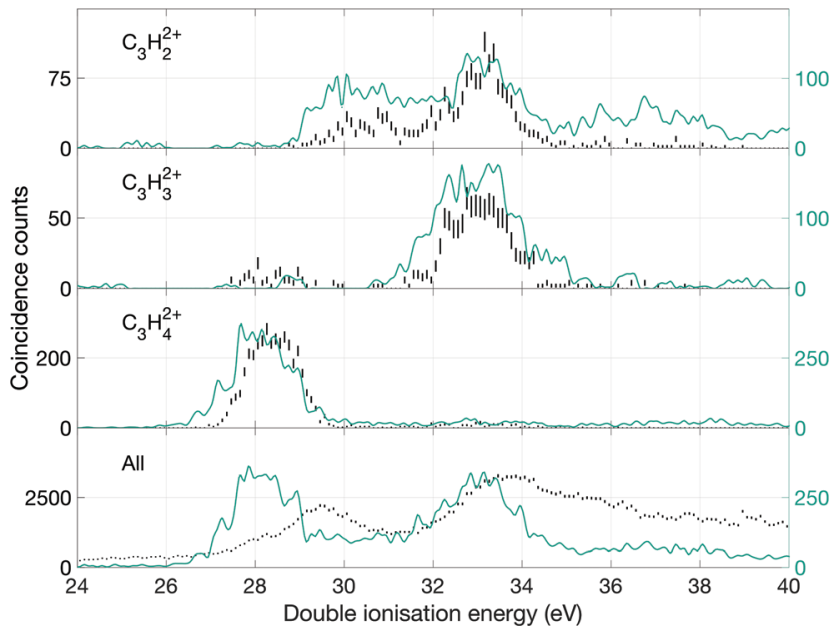

Fig. 9 Double ionization spectra based on electrons that were detected in coincidence with the charge retaining parent species or with charge retaining fragments which lost one or two hydrogen atoms. Because the detected species is doubly ionised and triple ionization is impossible at this photon energy, no other ions can be involved in these events. The green lines give the intensity profiles of the individual ions after correction for peak overlaps. The lowermost plot in black is the entire double ionization spectrum of all ions and the green line indicates the double ionization of the three peaks in green in the above panels, combined. All green spectra lack error bars because of the scaled number of counts with respect to the peak area.

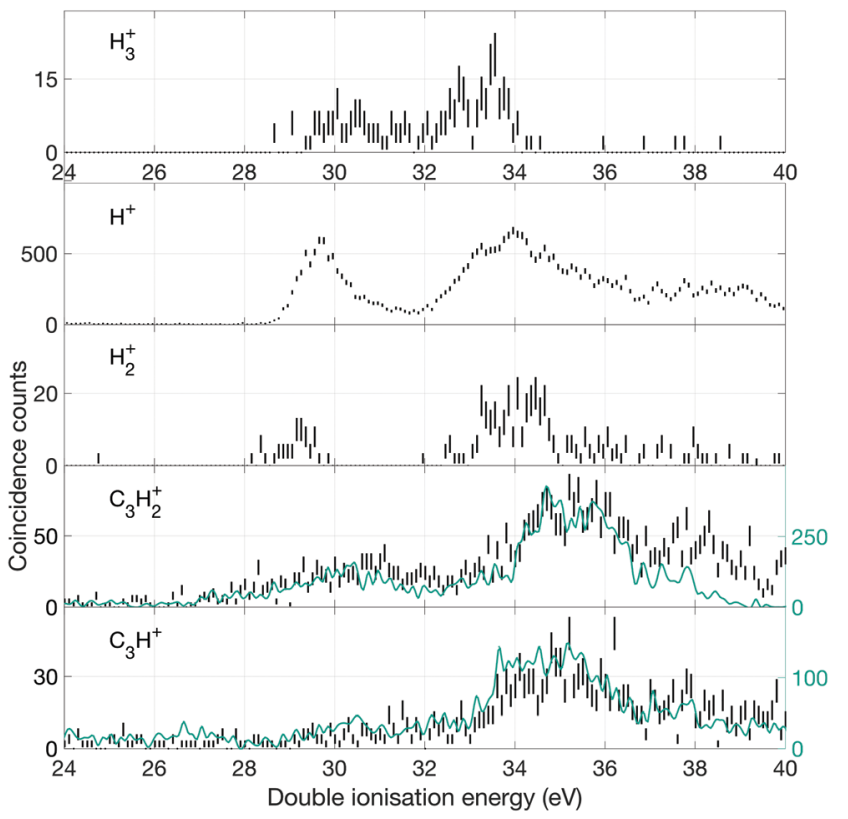

Fig. 10 Double ionization spectra based on two electrons detected in coincidence with a singly charged ion as specified for each spectrum. Both two-body and three-body fragmentation can contribute to these spectra, but two-body decays are probably dominant. The green line in the two lowermost panels are the number of counts found when correcting for a large peak overlap in $m / q=36-40$.

spectrum may be affected in a similar way. In order to demonstrate more realistic spectra for the doubly-charged ions we

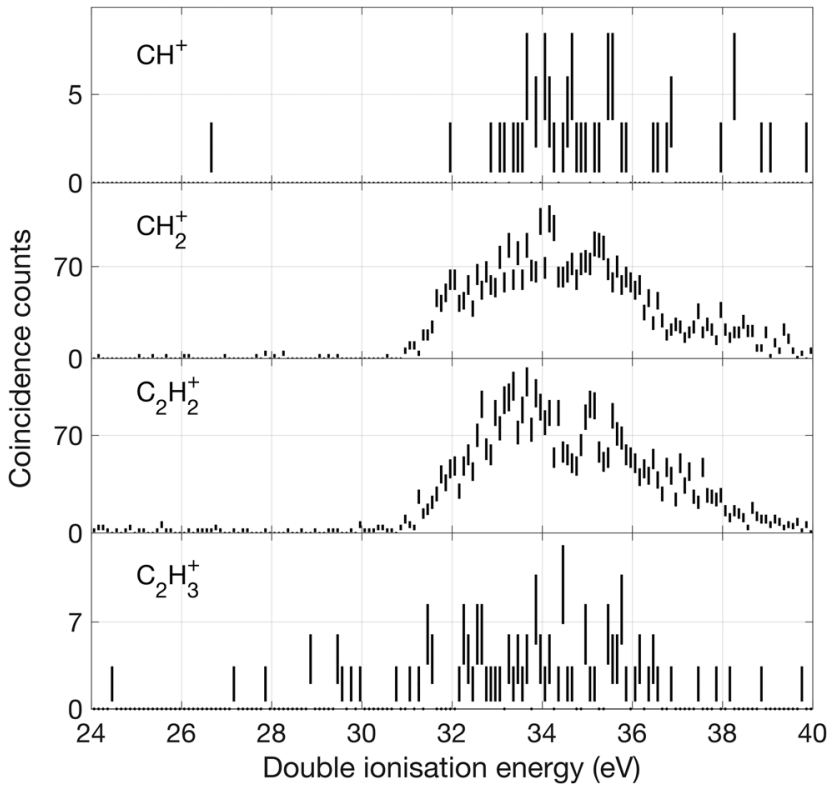

Fig. 11 Double ionization spectra for channels where a $\mathrm{C}-\mathrm{C}$ bond is broken, based on two electrons detected in coincidence with a singly charged ion as specified for each spectrum.

have carried out an iterative subtraction process, based on the estimated extent of peak overlap. The resulting spectra are shown in Fig. 9 in green together with the raw spectra in black.

Over the first $2 \mathrm{eV}$ above the onset of vertical double ionization at about $27 \mathrm{eV}$, the parent $\mathrm{C}_{3} \mathrm{H}_{4}{ }^{2+}$ ion remains stable on the mass spectrometer time scale. The lowest energy dissociation by charge separation is the slow metastable decay by $\mathrm{H}^{+}$ejection, with onset at $27.9 \pm 0.3 \mathrm{eV}$ and peak intensity at $29 \mathrm{eV} \pm 0.3 \mathrm{eV}$ shown in Fig. 12. The metastable signal appears only in a narrow range, presumably just above threshold, with a full width at half maximum (FWHM) of $0.7 \mathrm{eV}$, in line with the expected electron energy resolution at this ionization energy of ca $0.6 \mathrm{eV}$. This observation strengthens the view that a single vibrational level of the parent dication is responsible for the slow dissociation. Once the ionization energy exceeds $29 \mathrm{eV}$ formation of $\mathrm{H}^{+}+\mathrm{C}_{3} \mathrm{H}_{3}{ }^{+}$occurs rapidly and becomes the most probable dissociation pathway. However, at the same energy both $\mathrm{H}_{2}{ }^{+}$and $\mathrm{H}_{3}{ }^{+}$are formed with low intensity ( $c f$. Fig. 10). The three lowest panels in Fig. 12 all represent the process of $\mathrm{H}^{+}+$ $\mathrm{C}_{3} \mathrm{H}_{3}{ }^{+}$formation detected in different ways, and the differences between them illustrate the problems of poor statistics in fourfold coincidences and background subtraction of overlapping mass peaks.

Fig. 10 shows the yields of selected single ions in coincidence with electron pairs. For all the $\mathrm{C}_{3} \mathrm{H}_{\mathrm{n}}{ }^{+}$ions there is an overlap problem similar to that encountered for the doublycharged ions ( $c f$. Fig. 9) and we have compensated for it in a similar way. In this case, fourfold coincidence measurements give clear guidance on the extent of overlap and the necessary subtractions. Because of the threefold coincidence selection, the contributing ions may be formed by three-body as well as two-body dissociations, but the lowest energy pathways must be 
the two-body reactions forming $\mathrm{H}^{+}, \mathrm{H}_{2}{ }^{+}$and $\mathrm{H}_{3}{ }^{+}$with observed onset energies in the region of 28.5 to $29.5 \mathrm{eV}$. Where two-body reactions dominate, the spectra for $\mathrm{H}_{3}{ }^{+}$and $\mathrm{C}_{3} \mathrm{H}^{+}$and those of $\mathrm{H}_{2}{ }^{+}$and $\mathrm{C}_{3} \mathrm{H}_{2}{ }^{+}$should be the same. This is borne out by comparison of the spectra up to 35 or $36 \mathrm{eV}$, but at higher energy the different intensities indicate that three-body reaction releasing additional neutral fragments take over. The calculations of Mebel and Bandrauk ${ }^{18}$ indicate that barriers to the three hydrogen ion loss pathways should lie in the range of 28.84 to $29.03 \mathrm{eV}$, and their RRKM calculations predict that $\mathrm{H}_{3}{ }^{+}$ peak formation should occur at $29.53 \mathrm{eV}$. The calculated onsets and the peak production energy agree well with the observations in Fig. 10. However, further predictions based on the RRKM model assumption that all channels are in competition at all energies are not in line with our experimental data since the intensities of the channels leading to $\mathrm{H}^{+}$and $\mathrm{H}_{3}{ }^{+}$are not comparable at any energy. An alternative interpretation, that chimes with the "roaming" mechanism is that some of the initial population becomes isolated as a $\left[\mathrm{C}_{3} \mathrm{H}_{2}-\mathrm{H}_{2}\right]^{2+}$ complex, where the $\mathrm{H}_{2}$ can either escape or capture a proton. Such a mechanism would explain why $\mathrm{H}_{3}{ }^{+}$formation does not take over from $\mathrm{H}^{+}$production and why the intensities and spectra for $\mathrm{H}_{2}{ }^{+}$and $\mathrm{H}_{3}{ }^{+}$production are similar (cf. Fig. 10).

In the higher double ionization energy range, one of the main observations from our data is that the three-body products $\mathrm{H}^{+}+\mathrm{C}_{3} \mathrm{H}_{2}{ }^{+}+\mathrm{H}$ take over completely from $\mathrm{H}^{+}+\mathrm{C}_{3} \mathrm{H}_{3}{ }^{+}$at energies above $34 \mathrm{eV}$, as demonstrated by the spectra in Fig. 12 . The most natural explanation for that is that this is a sequential

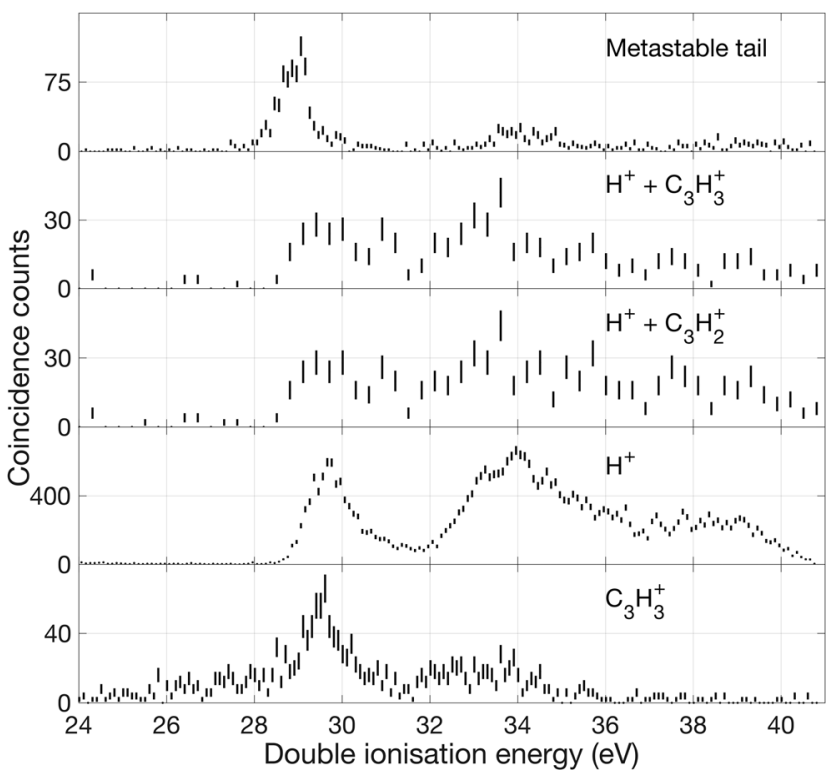

Fig. 12 Double ionization spectra based on electrons that were detected in coincidence with ions as specified in the figure. The uppermost spectrum represents decay events detected in the metastable tail shown in Fig. 7. The next lower spectra are derived from selection of events with two ions such as, $\mathrm{H}^{+}$and $\mathrm{C}_{3} \mathrm{H}_{3}{ }^{+}$, and two electrons, eeii. The lower spectra are from events with one ion $\mathrm{H}^{+}$or $\mathrm{C}_{3} \mathrm{H}_{3}{ }^{+}$, respectively, and two electrons, eei. One coincident count is equivalent to two electrons being detected in the same event. decay; $\mathrm{C}_{3} \mathrm{H}_{3}{ }^{+}$ions with sufficient internal energy (8 eV initially) dissociate further by $\mathrm{H}$ atom loss. Another very interesting channel is the production of $\mathrm{H}_{2}{ }^{+}+\mathrm{C}_{3} \mathrm{H}_{2}{ }^{+}$, which according to the eei data given in Fig. 10 occurs weakly between 28 and $30 \mathrm{eV}$, then more strongly above $32 \mathrm{eV}$. Formation near $29 \mathrm{eV}$ is possible only for the cyclic form of $\mathrm{C}_{3} \mathrm{H}_{2}{ }^{+}$(thermochemical threshold $25.5 \mathrm{eV}$ ) with a sensible kinetic energy release. Both the cyclic and perhaps more naturally produced linear $\mathrm{C}_{3} \mathrm{H}_{2}{ }^{+}$ ion (threshold $27.7 \mathrm{eV}$ ) can be formed at $32 \mathrm{eV}$, which is the energy where the second main double ionization band begins (cf. Fig. 2). According to a recent femtosecond laser pump-probe study of the roaming mechanism for $\mathrm{H}_{3}{ }^{+}$formation from doubly ionised methanol, ${ }^{19}$ the two exit channels giving $\mathrm{H}_{3}{ }^{+}$and $\mathrm{H}_{2}{ }^{+}$are both ultrafast and in competition. If that is also true for allene, the channel forming $\mathrm{H}_{3}{ }^{+}$must have entropic or related factors in its favour, as it takes over completely from $\mathrm{H}_{2}{ }^{+}$formation in the second part of the first main double ionization band (cf. Fig. 10). It is also striking that the charge retaining channel $\mathrm{H}_{2}+\mathrm{C}_{3} \mathrm{H}_{2}{ }^{2+}$ appears at $29.6 \pm 0.3 \mathrm{eV}$ ( $c f$. Fig. 9), near the energy where $\mathrm{H}_{2}{ }^{+}$ ceases to be produced (Fig. 10) and close to its calculated asymptote. This suggests that charge separation and charge retention by the heavier fragment are also in competition at this point.

There is an apparent reappearance of intensity for $\mathrm{H}^{+}+$ $\mathrm{C}_{3} \mathrm{H}_{3}{ }^{+}$peaking at around $34 \mathrm{eV}$ ( $c f$. Fig. 12) in the second double ionization band. In this range both the cyclic and linear isomers of the ion may be formed and charge-retaining formation of both $\mathrm{C}_{3} \mathrm{H}_{3}{ }^{2+}$ and $\mathrm{C}_{3} \mathrm{H}_{2}{ }^{2+}$ appear to compete strongly in the same energy range. As can be seen from Fig. 11, this is also the energy range where the charge-separating $\mathrm{C}-\mathrm{C}$ bondbreaking reaction giving rise to $\mathrm{CH}_{2}^{+}+\mathrm{C}_{2} \mathrm{H}_{2}{ }^{+}$becomes intense. It is slightly surprising that so many very different dissociation routes can all compete in the same energy range with comparable intensities in all the channels.

The appearance energies of the different sets of products determined from the experimental spectra in Fig. 9-11, are

Table 3 Double ionization of allene at $40.8 \mathrm{eV}$ photon energy in comparison to thermodynamic thresholds and theoretical predictions by Mebel and Bandrauk (M\&B). ${ }^{18}$ The uncertainties in the observed values are a consequence of the kinetic energy resolution of the electron spectrometer used

\begin{tabular}{|c|c|c|c|c|}
\hline \multirow[b]{2}{*}{ Channel } & \multicolumn{2}{|c|}{$0 \mathrm{~K}$ threshold $(\mathrm{eV})$} & \multicolumn{2}{|c|}{ Appearance energy (eV) } \\
\hline & Therm. & Calc. $(\mathrm{M} \& \mathrm{~B})^{18}$ & Calc. $(\mathrm{M} \& \mathrm{~B})^{18}$ & Obs. $(\mu s)$ \\
\hline $\mathrm{H}^{+}+\mathrm{C}_{3} \mathrm{H}_{3}^{+}$ & $\begin{array}{l}\text { Cyclic } 24.9 \\
\text { Linear } 26.0\end{array}$ & $\begin{array}{l}24.95 \\
26.2\end{array}$ & 28.8 & $28.9 \pm 0.3$ \\
\hline $\mathrm{H}^{+}+\mathrm{C}_{3} \mathrm{H}_{2}^{+}+\mathrm{H}$ & $\begin{array}{l}\text { Cyclic } 28.2 \\
\text { Linear } 30.3\end{array}$ & & & $32 \pm 0.3$ \\
\hline $\begin{array}{l}\mathrm{H}^{+}+\mathrm{C}_{3} \mathrm{H}^{+}+\mathrm{H}_{2} \\
\mathrm{H}_{2}^{+}+\mathrm{C}_{3} \mathrm{H}_{2}^{+}\end{array}$ & $\begin{array}{l}30.3 \\
\text { Cyclic } 25.5\end{array}$ & 27.7 & & $32.8 \pm 0.2$ \\
\hline $\mathrm{H}_{2}^{+}+\mathrm{C}_{3} \mathrm{H}^{+}+\mathrm{H}$ & $\begin{array}{l}\text { Linear } 27.7 \\
32.1\end{array}$ & 27.9 & 29.44 & \\
\hline $\mathrm{H}_{3}^{+}+\mathrm{C}_{3} \mathrm{H}^{+}$ & 25.9 & 26.0 & 29.03 & $29 \pm 0.3$ \\
\hline $\mathrm{CH}_{2}^{+}+\mathrm{C}_{2} \mathrm{H}_{2}^{+}$ & 26.1 & 26.2 & 30.8 (vinylidene) & $31 \pm 0.3$ \\
\hline $\begin{array}{l}\mathrm{CH}^{+}+\mathrm{C}_{2} \mathrm{H}_{3}^{+} \\
\mathrm{C}_{3} \mathrm{H}_{4}{ }^{2+}\end{array}$ & 26.3 & adb. 25.84 & 30.36 & $32.1 \pm 0.2$ \\
\hline $\begin{array}{l}\mathrm{C}_{3} \mathrm{H}_{3}{ }^{2+}+\mathrm{H} \\
\mathrm{C}_{3} \mathrm{H}_{2}{ }^{2+}+\mathrm{H}_{2}\end{array}$ & & $\begin{array}{l}\text { vert. } 28.05 \\
\text { c-30.55 } \\
29.92,29.39\end{array}$ & & $\begin{array}{l}27.5 \pm 0.3 \\
32.1 \pm 0.2 \\
29.6 \pm 0.3\end{array}$ \\
\hline
\end{tabular}


listed in Table 3 together with thermodynamic thresholds and theoretical predictions. For all the thermodynamic $0 \mathrm{~K}$ thresholds for ions formed with no internal energy or kinetic energy, heats of formation are taken from the NIST database or are estimated by combining known thermodynamic data with theoretical calculations (e.g. from Mebel and Bandrauk (M\&B) ${ }^{18}$ ). We note that the ions of mass 39,38 and possibly 37 can have either cyclic or linear structures, with significantly different heats of formation (the heats of formation for mass 37 is uncertain). The Mebel and Bandrauk-calculated thresholds are taken from their Fig. 2, with some degree of ambiguity.

\section{Conclusions}

Using multi-particle coincidence experiments we have obtained single-photon double and triple ionization spectra of allene, and have determined how dissociation of the doubly charged ions depends on the ionization energy. New high-level calculations confirm that adiabatic double ionization would require isomerization to a different structure, not accessible to vertical ionization processes. The calculations allow substructure in the first double ionization band to be assigned to different electronic states of the dications. Double ionization of allene by Auger decay of C1s hole states is found to populate different spectra of dication states according to location of the vacancy on either the central or an outer $\mathrm{C}$ atom.

Triple ionization of allene by three routes: valence ionization at $100 \mathrm{eV}$, double Auger decay of C1s holes and Auger decay of a C1s core-valence doubly ionised intermediate state yield different spectra, but all exhibit an onset of triple ionization at approximately $50 \mathrm{eV}$, in line with predictions available in the literature.

Eight significant decay pathways for dissociative decay of nascent allene dications have been identified and the energy dependence of their relative intensities is reported. At the lowest energies there is evidence that formation of $\mathrm{H}_{2}{ }^{+}+$ $\mathrm{C}_{3} \mathrm{H}_{2}{ }^{+}$and $\mathrm{H}_{3}{ }^{+}+\mathrm{C}_{3} \mathrm{H}^{+}$are in competition with each other and possibly with the charge-retaining $\mathrm{H}+\mathrm{C}_{3} \mathrm{H}_{3}{ }^{2+}$ channel, but not with charge separation to $\mathrm{H}^{+}+\mathrm{c}_{-} \mathrm{C}_{3} \mathrm{H}_{3}{ }^{+}$. This finding supports the roaming mechanism, already proposed, where an $\mathrm{H}_{2}$ molecule becomes partially detached from the heavy residual molecular dication.

For the decay to $\mathrm{H}^{+}+\mathrm{c}_{-} \mathrm{C}_{3} \mathrm{H}_{3}{ }^{+}$, we have confirmed the existence of a slow metastable decay happening in a narrow energy range just above threshold. This decay is well characterised as a single exponential with a mean lifetime determined as $130.5 \pm 9.9 \mathrm{~ns}$. We suspect that a single vibrational level of the parent dication may be involved and might be identified by future calculations.

\section{Author contributions}

R. F. and J. H. D. E. devised the research, V. I., M. W., E. O., R. J. S., U. M., E. F., M. F., S. S. and R. F. participated in the conduction of the experimental research, A. J. S. and F. D. carried out the theoretical calculations partially advised by J. M. D., V. I. and J. H. D. E. performed the data analysis, V. I., A. S., F. D., J. H. D. E. and R. F. wrote the first version of the paper and all authors discussed the results and commented on the manuscript.

\section{Conflicts of interest}

There is no conflict of interest.

\section{Acknowledgements}

This work has been financially supported by the Swedish Research Council (VR) and the Knut and Alice Wallenberg Foundation, Sweden. A. J. S. thanks the EPSRC Centre for Doctoral Training in Synthesis for Biology and Medicine for a studentship (EP/L015838/1). We thank the Helmholtz Zentrum Berlin for the allocation of synchrotron radiation beam time and the staff of BESSY-II for smooth operation of the storage ring in single-bunch mode. The research leading to these results has received funding from the European Union's Horizon 2020 research and innovation programme under grant agreement No. 730872 .

\section{References}

1 R. I. Kaiser, D. Stranges, Y. T. Lee and A. G. Suits, Astrophys. J., 1997, 477, 982.

2 B. M. Jones, F. Zhang, R. I. Kaiser, A. Jamal, A. M. Mebel, M. A. Cordiner and S. B. Charnley, Proc. Natl. Acad. Sci. U. S. A., 2011, 108, 452-457.

3 R. Ruiterkamp, S. B. Charnley, H. M. Butner, H.-C. Huang, S. Rodgers, Y.-J. Kuan and P. Ehrenfreund, Astrophys. Space Sci., 2007, 310, 181-188.

4 R. Stockbauer, K. E. McCulloh and A. C. Parr, Int. J. Mass Spectrom. Ion Phys., 1979, 31, 187-189.

5 C. Baker and D. W. Turner, J. Chem. Soc. D, 1969, 480-481.

6 R. K. Thomas and H. W. Thompson, Proc. R. Soc. London, Ser. A, 1974, 339, 29-36.

7 G. Bieri, F. Burger, E. Heilbronner and J. P. Maier, Helv. Chim. Acta, 1977, 60, 2213-2233.

8 J. Dannacher and J. Vogt, Helv. Chim. Acta, 1978, 61, 361-372.

9 M. H. Garner, R. Hoffmann, S. Rettrup and G. C. Solomon, ACS Cent. Sci., 2018, 4, 688-700.

10 C. H. Hendon, D. Tiana, A. T. Murray, D. R. Carbery and A. Walsh, Chem. Sci., 2013, 4, 4278-4284.

11 E. Soriano and I. Fernandez, Chem. Soc. Rev., 2014, 43, 3041-3105.

12 S. R. Andrews, D. E. Parry, M. Vairamani and F. M. Harris, J. Chem. Soc., Faraday Trans., 1992, 88, 3403-3407.

13 S. R. Andrews, D. E. Parry and F. M. Harris, J. Chem. Soc., Faraday Trans., 1995, 91, 1181-1183.

14 K. Hoshina, H. Kawamura, M. Tsuge, M. Tamiya and M. Ishiguro, J. Chem. Phys., 2011, 134, 064324. 
15 H. Xu, C. Yao, G. Xu, Z. Wang and H. Jin, Combust. Flame, 2013, 160, 1333-1344.

16 K. Hoshina, Y. Furukawa, T. Okino and K. Yamanouchi, J. Chem. Phys., 2008, 129, 104302.

17 N. Iwamoto, C. J. Schwartz, B. Jochim, K. Raju, P. P. Feizollah, J. Napierala, T. Severt, S. Tegegn, A. Solomon and S. Zhao, et al., J. Chem. Phys., 2020, 152, 054302.

18 A. M. Mebel and A. D. Bandrauk, J. Chem. Phys., 2008, 129, 224311.

19 E. Livshits, I. Luzon, K. Gope, R. Baer and D. Strasser, Commun. Chem., 2020, 3, 1-6.

20 J. H. D. Eland, O. Vieuxmaire, T. Kinugawa, P. Lablanquie, R. I. Hall and F. Penent, Phys. Rev. Lett., 2003, 90, 053003.

21 J. H. D. Eland and R. Feifel, Chem. Phys., 2006, 327, 85.

22 J. H. D. Eland and R. Feifel, J. Chem. Phys., 2006, 125, 194318.

23 S. Plogmaker, P. Linusson, J. H. D. Eland, N. Baker, E. M. J. Johansson, H. Rensmo, R. Feifel and H. Siegbahn, Rev. Sci. Instrum., 2012, 83, 013115.

24 Y. A. Kruglyak, H. Preuss and R. Janoschek, Theor. Exp. Chem., 1973, 7, 241.

25 F. Neese, Rev. Comput. Mol. Sci, 2018, 8, 4.

26 F. Neese, Phys. Chem. Chem. Phys., 2005, 7, 3297.
27 C. Angeli, R. Cimiraglia, S. Evangelisti, T. Leininger and J. P. Malrieu, J. Chem. Phys., 2001, 114, 10252.

28 M. W. Wong and L. Radom, J. Mol. Struct., 1989, 198, 391-402.

29 O. Travnikova, PhD thesis, Acta Universitatis Upsaliensis, 2008.

30 A. Hult Roos, PhD thesis, The University of Gothenburg, The Faculty of Science, 2019.

31 H. Hogreve, J. Chem. Phys., 1995, 102, 3281-3291.

32 A. Faibis, E. P. Kanter, L. M. Tack, E. Bakke and B. J. Zabransky, J. Phys. Chem., 1987, 91, 6445-6447.

33 P. R. Taylor, J. M. L. Martin, J. P. Francois and R. Gijbels, J. Phys. Chem., 1991, 95, 6530-6534.

34 S. Diaz-Tendero, G. Sánchez, P.-A. Hervieux, M. Alcam and F. Martn, Braz. J. Phys., 2006, 36, 529-533.

35 T. A. Field and J. H. D. Eland, Chem. Phys. Lett., 1993, 211, 436-442.

36 M. Barber and K. R. Jennings, Zeitschrift für Naturforschung A, 1969, 24, 134-138.

37 D. Manura and D. Dahl, SIMION 8.0/8.1 User Manual: Covering SIMION Version 8.1.0.31, Scientific Instrument Services, Incorporated, 2011. 